\title{
The Mechanism of the Helix-Sense Inversion of Polyaspartates as Revealed by the Study of Model Block Copolymers
}

\author{
Akinobu UshiYama, Hidemine FurUYa,${ }^{\dagger}$ Akihiro ABE, ${ }^{*}$ \\ and Toshimasa YAMAZAKI** \\ Department of Organic and Polymeric Materials, Tokyo Institute of Technology, \\ 2-12-1 Ookayama, Meguro-ku, Tokyo 152-8552, Japan \\ * Department of Applied Chemistry, Tokyo Institute of Polytechnics, \\ 1583 Iiyama, Atsugi, Kanagawa 243-0297, Japan \\ ${ }^{* *}$ National Institute of Agrobiological Sciences, \\ 2-1-2 Kannondai, Tsukuba, Ibaraki 305-8602, Japan
}

(Received January 10, 2002; Accepted April 22, 2002)

\begin{abstract}
For the purpose of elucidating the mechanism of the helix-sense inversion phenomena characteristic of poly(aspartic ester)s, we synthesized two diblock copolymers comprising poly( $\beta$-phenethyl L-aspartate) (PA) and poly $(\gamma$ benzyl L-glutamate) (BG) blocks designated as BG-PA and PA-BG in the order from the C-terminus to the N-terminus. A triblock copolymer sample such as poly( $\gamma$-benzyl L-glutamate)- $b$-poly( $\beta$-phenethyl L-aspartate)- $b$-poly $(\gamma$-benzyl Lglutamate) (BG-PA-BG) was also prepared. The screw sense of $\alpha$-helical block copolymers was investigated in 1,1,2,2tetrachloroethane (TCE) solution by the ${ }^{1} \mathrm{H}$ nuclear magnetic resonance (NMR) and circular dichroism techniques as a function of temperature. For the BG-PA diblock copolymer, the helix-sense of the PA block was completely reversed from right- to left-handed at $100^{\circ} \mathrm{C}$ on heating. The thermal behaviors of triblock copolymer BG-PA-BG was found to be very similar to those of the poly $(\gamma$-benzyl L-glutamate) (BG) homopolymer, indicating that the reversal of the screwsense was entirely restrained. From these observations, we have concluded that during heating, the helix-helix transition proceeds predominantly from the $\mathrm{N}$-terminus in the $\alpha$-helical polyaspartate system.
\end{abstract}

KEY WORDS Poly( $\beta$-phenethyl L-aspartate) / Poly ( $\gamma$-benzyl L-glutamate $) /$ Block Copolymer / Helix-Sense Inversion / ${ }^{1} \mathrm{H}$ Nuclear Magnetic Resonance (NMR) / Circular Dichroism /

It is well known that poly(aspartic acid ester)s $\left[\mathrm{NHCH}\left(\mathrm{CH}_{2} \mathrm{COOR}\right) \mathrm{CO}\right]_{n^{-}}$form either the right- or left-handed $\alpha$-helical conformation depending on the chemical structure of the $\mathrm{R}$ group, temperature or solvent. $^{1-7}$ While poly $(\beta$-benzyl L-aspartate) (BA) and $\operatorname{poly}(\beta$-methyl L-aspartate) form the left-handed $\alpha$ helix in chloroform, the right-handed helix is more popular among polyaspartates having $\mathrm{R}=$ ethyl or phenethyl group. An inversion of the helix sense in the polyaspartate copolymer system comprising nitrobenzyl and benzyl residues at the ester terminal has been extensively investigated by Goodman et al. in 1960 's. ${ }^{8-10}$ Later the other examples such as random copolymers, $\operatorname{copoly}(\beta$-alkyl L-aspartate- $\beta$-benzylL-aspartate)s and diblock copolymers, $\operatorname{poly}(\gamma$-benzyl L-glutamate)- $b$-poly( $\beta$-benzyl L-aspartate) (BG- $b$-BA) have been respectively studied by Tsujita et al. ${ }^{11,12}$ and by Paolillo et al. ${ }^{13}$ The optical rotatory dispersion, circular dichroism (CD) $)^{11,12}$ and ${ }^{1} \mathrm{H}$ nuclear magnetic resonance (NMR) ${ }^{13}$ measurements in dilute solution suggested that the screw-sense of the $\alpha$-helical aspartate residues could be affected by the composition as well as the order of monomer sequence along the copolymer backbone.

\footnotetext{
${ }^{\dagger}$ To whom correspondence should be addressed.
}

The helix-sense inversion behaviors of $\operatorname{poly}(\beta$ phenethyl L-aspartate) (PA) have been studied by Uematsu and his collaborators. While Sasaki et al. ${ }^{14}$ have found that PA exhibits an irreversible helix-sense inversion from the right-handed $\alpha$-helix to the left-handed $\pi$-helix in the solid state at some elevated temperature, Watanabe et al. ${ }^{15,16}$ have observed a thermally reversible helix-sense inversion of $\operatorname{poly}(\beta$-phenylpropyl L-aspartate) in the solid film. Toriumi et al. ${ }^{17}$ have reported a transition from the right- to left-handed $\alpha$-helix in chloroform by addition of a small amount of denaturant acid. In our previous studies on PA, ${ }^{18-23}$ we have found that the transition between the right- and lefthanded $\alpha$-helices takes place reversibly at around $85^{\circ} \mathrm{C}$ in an isotropic solution as well as in the lyotropic liquid crystalline state. In the presence of denaturant acid, PA also exhibits a transformation from the right- to left-handed $\alpha$-helix at lower temperatures. Deuterium NMR studies using ${ }^{2} \mathrm{H}$-labeled PA have revealed that the orientational order of the backbone is maintained in the lyotropic liquid crystalline state during the helixsense reversal, suggesting that a certain elaborate mechanism is involved in these transitions. The primary cause of these highly cooperative helix-sense reversals was found to reside in a small free-energy difference 
in the side-chain conformation flanking the right- and left-handed $\alpha$-helical poly(amino acid) backbone.

In this study, we have attempted to investigate more details of the mechanism of the helix-sense inversion of PA by preparing block copolymers. Diblock and triblock copolymers consisting of PA and BG blocks were synthesized. The helix sense of the PA block was identified by the ${ }^{1} \mathrm{H}$ NMR and CD measurements. $\alpha$ Helical BG is known to be stable in the right-handed form over the temperature range employed in this experiment. The helix-sense inversion behavior of the PA block may be affected by the presence of a BG block, on either N-terminus or C-terminus, in the same molecule. Studies of these block copolymers should provide some insight into the mechanism of the helixsense inversion of the PA system.

\section{EXPERIMENTAL}

\section{Samples}

Block copolymer samples were synthesized by sequential polymerization of $N$-carboxy $\beta$-phenethyl L-aspartate anhydride and $N$-carboxy $\gamma$-benzyl Lglutamate anhydride. ${ }^{13,23-25}$ The first block was prepared by polymerization in chloroform by using diethylamine as the initiator, the molar ratio of the anhydride to the initiator, $\mathrm{A} / \mathrm{I}$, being set equal to $100 .{ }^{25}$ The polymerization for the initial sequence was performed for 2 days at room temperature. For the completion of the polymerization, the unreacted anhydride remained in solution was checked by NMR measurement. $\mathrm{A} \mathrm{CHCl}_{3}$ solution of the second anhydride was added to the flask without intermediate isolation of the first block and the polymerization was allowed to proceed for another 3 days to completion. For the triblock copolymer, a solution of the third anhydride was subsequently added, and the polymerization was carried out for another 3 days. The block copolymers obtained were purified by repeated precipitation from chloroform in a large volume of methanol.

\section{Characterization}

Chemical structures of the diblock copolymers (BG$\mathrm{PA}$ and PA-BG) and the triblock copolymer (BG-PABG) prepared in this study are shown in Figure 1. The compositions of the blocks correspond to the proportions of anhydrides used in preparing the block copolymer. The copolymer composition (i.e., block lengths) was estimated by integration of the characteristic ${ }^{1} \mathrm{H}$ NMR peaks assigned to the individual PA and BA blocks. The degrees of polymerization were found to be nearly equivalent for all PA and BG blocks prepared. The molecular weights of the sample were estimated
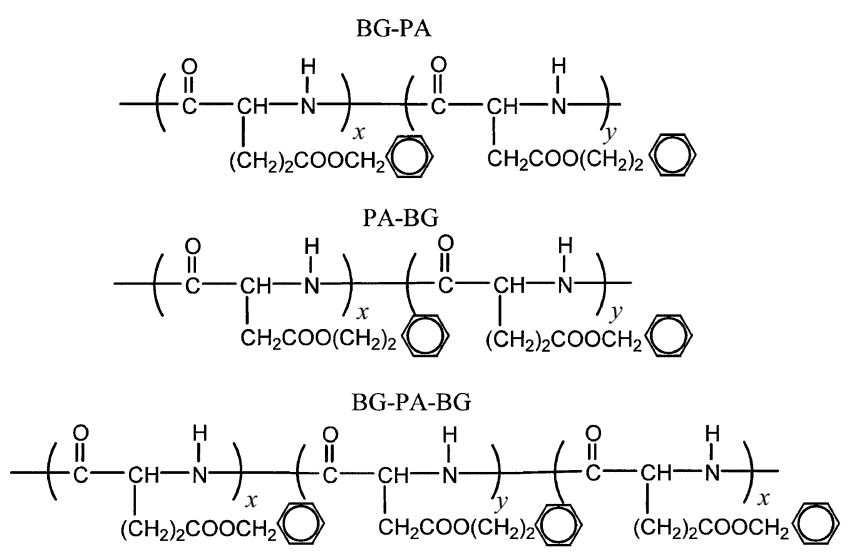

Figure 1. Chemical structures of the block copolymer samples used in the present experiment.

from the intrinsic viscosities $[\eta]$ measured in a mixed solvent (dichloroacetic acid $\left.(2 \% \mathrm{v} / \mathrm{v}) / \mathrm{CHCl}_{3}\right)$ at $25^{\circ} \mathrm{C}$ according to Hayashi et al.'s relation ${ }^{26}$ reported for BA homopolymer in the same solvent. The $[\eta]$ values $(\mathrm{dL}$ $\mathrm{g}^{-1}$ ) and the molecular weights of the sample are as follows: BG-PA, 0.32, $2.5 \times 10^{4}$; PA-BG, $0.40,3.0 \times 10^{4}$; BG-PA-BG, $0.40,3.0 \times 10^{4}$.

The Edman degradation method provides information regarding the type of amino acids constituting the $\mathrm{N}$-terminal sequence up to four residues. Aspartic and glutamic acids were respectively found to be the only products obtained from the BG-PA and PA-BG copolymers. The chemical structures of BG-PA and PA-BG block copolymers were thus confirmed.

\section{Measurements}

${ }^{1} \mathrm{H}$ NMR spectra were recorded on a JEOL JNMGSX-500 spectrometer operated at $500 \mathrm{MHz}$. Measurements were carried out at temperatures ranging from 30 to $110^{\circ} \mathrm{C}$ in TCE solution $(c a .1 \% \mathrm{w} / \mathrm{v})$.

CD spectra were recorded on a Jasco J-720WI spectrometer using a $0.1 \mathrm{~mm}$ jacketed quartz cell over the temperature range $30-110^{\circ} \mathrm{C}$. The measurements were carried out in the wavelength region between 200 and $260 \mathrm{~nm}$.

\section{RESULTS AND DISCUSSION}

\section{Helix-Sense Inversion of Diblock Copolymers}

The chemical shifts of $\mathrm{NH}$ and $\mathrm{C}^{\alpha} \mathrm{H}$ protons vary sensitively with the screw sense of the $\alpha$-helix. ${ }^{27}$ To avoid the uncertainty arising from the overlap between the $\mathrm{C}^{\alpha} \mathrm{H}$ and the $\mathrm{C}^{\varepsilon} \mathrm{H}$ resonance peaks, the $\mathrm{NH}$ proton signal was extensively used to identify the helix sense of the PA block. The NH signals appear at 8.2 and $8.8 \mathrm{ppm}$, respectively, for the right- and left-handed $\alpha$ helix. Figure 2 shows the variation of (a) NH signals of BG-PA and (b) those of PA-BG copolymers with tem- 


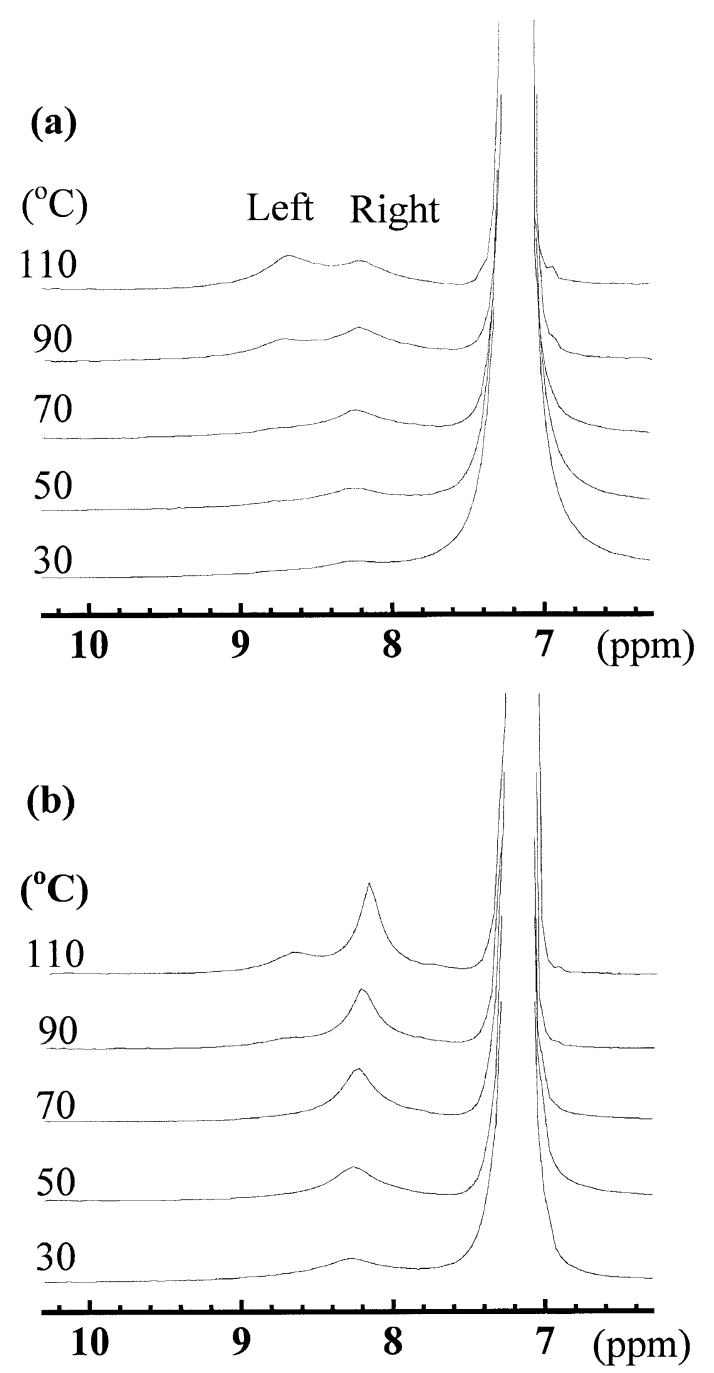

Figure 2. NH proton signals of diblock copolymers observed at various temperatures: (a) BG-PA and (b) PA-BG.

perature. For the BG-PA copolymer, the intensity of the $\mathrm{NH}$ peak situated at $8.2 \mathrm{ppm}$ decreases with increasing temperature. At $70^{\circ} \mathrm{C}$, another $\mathrm{NH}$ signal begins to appear at $8.8 \mathrm{ppm}$ and its intensity tends to be enhanced as temperature increases. Above $100^{\circ} \mathrm{C}$, the intensity of the NH signal at $8.2 \mathrm{ppm}$ becomes almost equivalent to that of the peak at $8.8 \mathrm{ppm}$. The NH signal remaining at $8.2 \mathrm{ppm}$ at higher temperatures may be attributable to the BG block, whose helix sense should be kept invariable (right-handed) under the experimental condition. The results described above lead to a scheme in which the helix-sense inversion of the PA part has been completed at certain temperature while the screw sense of the BG part remains unchanged. For the PA-BG sample, the NH signal of lower chemical shift $(8.8 \mathrm{ppm})$ becomes weakly visible above $100^{\circ} \mathrm{C}$ (Figure 2b). Although a minor fraction of PA block may exist in the left-handed form at higher temperatures, the ratio of the intensities of the two NH peaks at 8.2 and $8.8 \mathrm{ppm}$ is far from 1 to 1 . The NH signal ( $7.8 \mathrm{ppm})$ characteristic of
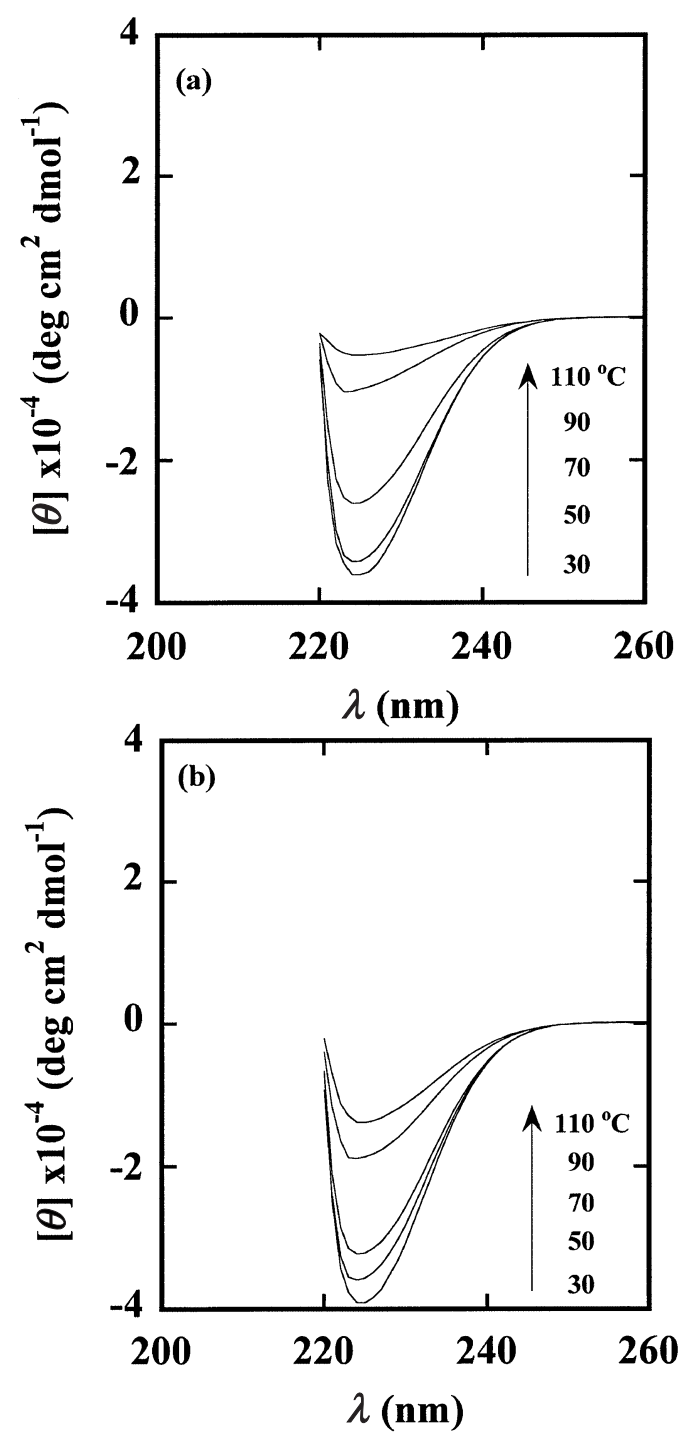

Figure 3. Circular dichroism spectra obtained at several temperatures: (a) BG-PA and (b) PA-BG.

the random-coil conformation could not be detected at any intermediate stage of the helix-sense inversion of the PA segments involved in the diblock copolymers.

Figure 3 shows the temperature dependence of $C D$ spectra in TCE for samples (a) BG-PA and (b) PA$\mathrm{BG}$. The wavelength region is effectively limited within 200-210 $\mathrm{nm}$ due to the intense absorption originating from the solvent (TCE). Both diblock copolymers exhibit a minimum at $222 \mathrm{~nm}$. The CD spectra of PA homopolymer is known to change its sign from negative to positive on heating in conjugation with the transformation of the helical sense from right- to left-handed. In Figure 3, the magnitude of $[\theta]_{222}$ tends to diminish with an increase in temperature in both diagrams (a) and (b). When the helix-sense inversion of the PA block is completed in these copolymers, the value of $[\theta]_{222}$ should reach zero due to the compensation of the contribution from the two oppositely twisted $\alpha$-helical blocks. Figure 4 indicates the variation of the $[\theta]_{222}$ 


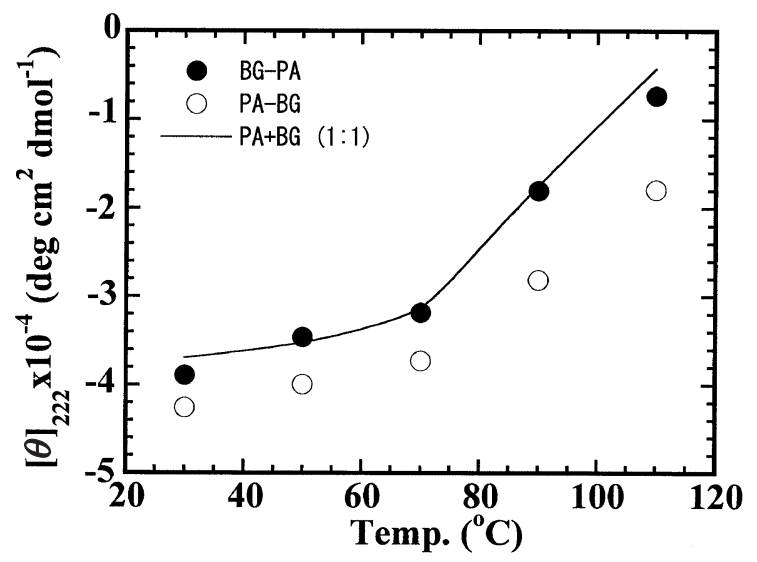

Figure 4. Variation of ellipticity at $222 \mathrm{~nm},[\theta]_{222}$, as a function of temperature: BG-PA (filled circles), PA-BG (open circles), and an equimolar mixture of homopolymers, $\mathrm{PA}+\mathrm{BG}$ (solid curve).

value with temperature for both diblock copolymers: BG-PA (filled circles) and PA-BG (open circles). For comparison, the corresponding results obtained from an equimolar mixture of the parent homopolymers PA and BG are also included (solid curve). The magnitude of $[\theta]_{222}$ observed for BG-PA was found to be very close to those obtained from the mixture of PA and $\mathrm{BG}$ over the entire temperature range. The $[\theta]_{222}$ values of PA-BG were somewhat lower than those observed for both BG-PA and the homopolymer mixture. In all cases, the $[\theta]_{222} v s$. temperature curves change their slopes distinctly around $70^{\circ} \mathrm{C}$, indicating that the helix-helix transition for the PA block set in around this temperature. The fractions of the left-handed $\alpha$ helix estimated from the $[\theta]_{222}$ value at $110^{\circ} \mathrm{C}$ are $c a$. 98 and $60 \%$, respectively, for BG-PA and PA-BG. The results of $\mathrm{CD}$ measurements suggest that the transformation of the PA block from the right- to left-handed $\alpha$-helix is nearly completed at this temperature in the former system. These observations suggest a transition mechanism in which the helix-sense inversion proceeds preferably from the N-terminus of the PA chain at elevated temperatures.

\section{Helix Sense of Triblock Copolymer}

Figure 5 indicates the temperature dependence of the NH signal derived from the BG-PA-BG triblock copolymer. The chemical shift of the NH proton was found to remain invariant at $8.2 \mathrm{ppm}$ over the temperature range from $30-110^{\circ} \mathrm{C}$. The resonance peak becomes sharper and its intensity tends to be enhanced as a function of temperature due to the increased mobility (vibration and rotation) of the molecule. In these measurements, no trace of a signal could be detected at around $8.8 \mathrm{ppm}$. The results lead to a conclusion that a PA block capped by BG blocks at both terminals does not exhibit the helix-sense inversion under an ordinary

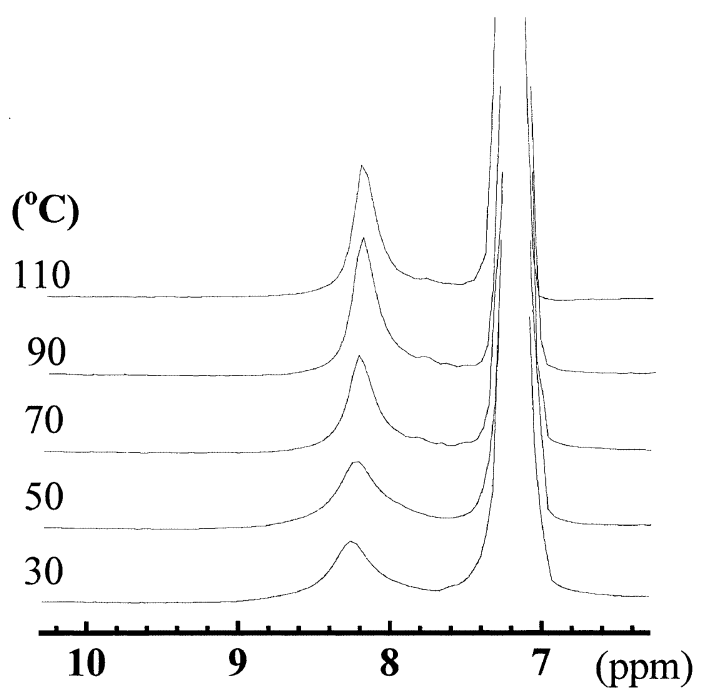

Figure 5. $\mathrm{NH}$ proton signals of triblock copolymer BG-PA-BG observed at various temperatures.

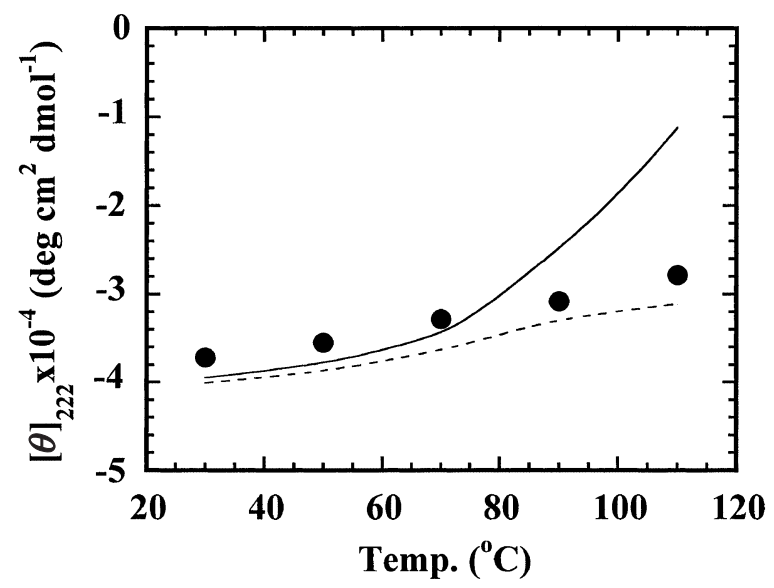

Figure 6. Variation of $[\theta]_{222}$ as a function of temperature for BG-PA-BG (filled circles), BG (broken curve), and a 1:2 mixture of PA and BG (solid curve).

condition.

In Figure 6, the $[\theta]_{222}$ values obtained for BG-PA$\mathrm{BG}$ are plotted against temperature (filled circles). The values of $[\theta]_{222}$ observed for BG homopolymer (broken curve) and a binary mixture comprising PA and $\mathrm{BG}$ in the 1:2 ratio (solid curve) are also shown for comparison. The values of $[\theta]_{222}$ for BG-PA-BG and $\mathrm{BG}$ tend to increase gradually with temperature in the range $30-110^{\circ} \mathrm{C}$. The mixture of $\mathrm{BG}$ and $\mathrm{PA}$ homopolymers follows a similar trend below $70^{\circ} \mathrm{C}$, but around this point, the slope of the solid curve becomes appreciably steeper, corresponding to the onset of the helixhelix transition of PA. In contrast, the conformation of the central PA block involved in the BG-PA-BG copolymer remains unchanged due to the restriction caused by the helical arrangement of the neighboring BG blocks situated on both sides. The difference between the triblock copolymer (filled circles) and the homopolymer mixture (solid curve) suggests that the helix-sense in- 
version (right to left) proceeds less likely from a middle portion of the PA block. In this respect, the results of the CD measurements were found to be quite consistent with the transition scheme derived from the ${ }^{1} \mathrm{H}$ NMR measurements.

\section{CONCLUSION}

In this work, three block copolymers such as BG-PA, PA-BG, and BG-PA-BG were prepared and conformational analyses were carried out in dilute TCE solution. Variation of the screw sense of $\alpha$-helix was followed by using the ${ }^{1} \mathrm{H}$ NMR and $\mathrm{CD}$ techniques. The results may be summarized as follows: (1) When a PA block is placed between two BG blocks (BG-PA-BG), the conformational transition of the PA block is totally prohibited even at high temperatures $\left(\sim 110^{\circ} \mathrm{C}\right),(2)$ When the $\mathrm{BG}$ block is connected to the $\mathrm{C}$-terminal side of the PA block in the diblock copolymer (i.e., BG-PA), the transformation of the $\alpha$-helical PA block takes place in the same temperature range $\left(>\sim 70^{\circ} \mathrm{C}\right)$ as those normally observed for the corresponding homopolymer PA, and (3) When the order of PA and BG blocks are reversed in the diblock copolymer (i.e., PA-BG), the rate of the helix-sense inversion of the PA block tends to be appreciably suppressed in comparison with the counterpart (BG-PA).

These experimental observations suggest that the inversion of the $\alpha$-helical screw sense predominantly proceeds from the $\mathrm{N}$-terminus of the PA molecule when the temperature becomes sufficiently high. More detailed studies on the mechanism of the helix-helix transition of polyaspartates are underway.

Acknowledgment. We would like to thank Dr. Yuji Nishiuchi of Peptide Institute, Inc., Protein Research Foundation for carrying out amino acid analyses of block copolymers.

\section{REFERENCES}

1. E. R. Blout and R. H. Karlson, J. Am. Chem. Soc., 80, 1259 (1958).

2. R. H. Karlson, K. S. Norland, G. D. Fasman, and E. R. Blout, J. Am. Chem. Soc., 82, 2268 (1960).
3. E. M. Bradbury, A. R. Downie, A. Elliott, and W. E. Hanby, Proc. Roy. Soc. London A, 259, 110 (1960).

4. E. M. Bradbury, B. G. Carpenter, and H. Goldman, Biopolymers, 6, 837 (1968).

5. E. M. Bradbury, B. G. Carpenter, and R. M. Stephens, Biopolymers, 6, 905 (1968).

6. M. Hashimoto and J. Aritomi, Bull. Chem. Soc. Jpn., 39, 2707 (1966).

7. M. Hashimoto and S. Arakawa, Bull. Chem. Soc. Jpn., 40, 1698 (1967)

8. M. Goodman, C. M. Deber, and A. M. Felix, J. Am. Chem. Soc., 84, 3773 (1962).

9. M. Goodman, A. M. Felix, C. M. Deber, A. R. Brause, and G. Schwartz, Biopolymers, 1, 371 (1963).

10. C. Toniolo, M. L. Falxa, and M. Goodman, Biopolymers, 6, 1579 (1968)

11. Y. Tsujita, N. Fujii, Y. Imoto, A. Takizawa, and T. Kinoshita, J. Polym. Sci., Polym. Chem. Ed., 22, 2533 (1984).

12. R. Nishizima, Y. Tsujita, and A. Takizawa, Polymer, 26, 379 (1985).

13. L. Paolillo, P. Temussi, E. Trivellone, E. M. Bradbury, and C. Crane-Robinson, Biopolymers, 10, 2555 (1971).

14. S. Sasaki, Y. Yasumoto, and I. Uematsu, Macromolecules, 14, 1797 (1981).

15. J. Watanabe, S. Okamoto, K. Satoh, K. Sakajiri, H. Furuya, and A. Abe, Macromolecules, 29, 7084 (1996).

16. K. Sakajiri, K. Satoh, K. Yoshioka, S. Kawauchi, and J. Watanabe, J. Mol. Struct., 477, 175 (1999).

17. H. Toriumi, N. Saso, Y. Yasumoto, S. Sasaki, and I. Uematsu, Polym. J., 11, 977 (1979).

18. A. Abe, S. Okamoto, N. Kimura, K. Tamura, H. Onigawara, and J. Watanabe, Acta Polym., 44, 54 (1993).

19. J. Watanabe, S. Okamoto, and A. Abe, Liq. Cryst., 15, 259 (1993).

20. S. Okamoto, H. Furuya, and A. Abe, Polym. J., 27, 746 (1995).

21. A. Abe, H. Furuya, and S. Okamoto, Biopolymers. Peptide Science, 43, 405 (1997).

22. A. Abe, H. Furuya, and S. Okamoto, Polym. Sci., Ser. A, 38, 317 (1996).

23. S. Okamoto, Ph. D. Dissertation in Polymer Chemistry, Tokyo Institute of Technology, 1995.

24. W. H. Daly and D. Poche, Tetrahedron Lett., 29, 5859 (1988).

25. J. C. Mitchell, A. E. Woodward, and P. Doty, J. Am. Chem. Soc., 79, 3955 (1957)

26. Y. Hayashi, A. Teramoto, K. Kawahara, and H. Fujita, Biopolymers, 8, 403 (1969).

27. E. M. Bradbury, B. G. Carpenter, C. Crane-Robinson, and H. Goldman, Macromolecules, 4, 557 (1971). 